\title{
Age-specific distribution of Human Papilloma Virus (HPV) mucosal infection among young females
}

\author{
Annalisa Pieralli*, Maria Grazia Fallani, Virginia Lozza, Serena Corioni, Manuela Longinotti, \\ Massimiliano Fambrini, Carlo Penna
}

Department of the Sciences for Woman's and Child's Health, University of Florence, Italy.

E-mail: ${ }^{*}$ pierallannalisa@virgilio.it

Received 30 May 2011; revised 5 July 2011; accepted 13 July 2011.

\begin{abstract}
The goal of our study was to describe the age-specific distribution of HPV genotypes and related disease among females under the age of 25 years. A prospective cohort study was carried out. We enrolled 85 young females aged 16 - 25 years ( 30 aged 16 - 19 and 55 aged 20 - 25 years) referred to our colposcopic unit after a repeated abnormal Pap smear result. Every patient underwent an HPV DNA testing, a colposcopy and eventually a cervical biopsy. Participants were proposed to follow-up or treatment on request. Treatment was performed by destructive or excisional laser $\mathrm{CO}_{2}$ therapy. Data were analyzed by Fisher's Exact test. The overall prevalence of low-risk HPV amounted to $80 \%$ among 16 - 19-year-old girls, while the overall prevalence of high-risk HPV was $85.5 \%$ among 20 25 -year-old patients. The univariate analysis of chosen characteristics of HPV-disease demonstrates the statistically significative difference of this infection between the two groups of age $(P<0.005)$. We observed a particular age-specific stratification of HPV genotypes and related disease, which appeared to be characterized by a cut-off at the age of 20 years. According to our data, cervical screening program in Italy seems to start later than the beginning of HPV-related pathology.
\end{abstract}

Keywords: HPV; Epidemiology; Adolescents; Genital Warts

\section{INTRODUCTION}

According to available data, genital infection with the Human Papillomavirus is highly prevalent in young female population from all countries and it is the most common sexually transmitted infection among sexually active girls [1-6]. Its prevalence in young women ranges from about 20 to $46 \%$ in various countries [7-9].

Sexually active adolescents show the highest HPV prevalence and incidence and over $50 \%-80 \%$ of them acquire the infection within two-three years of initiating sexual intercourse.

Data about the real distribution of HPV mucosal infection and related histological lesions in young women are scarce in literature. The actual knowledge of HPV-linked genital pathology and its natural history in adolescence principally derives from screening test results and not from clinical and histological findings, because care providers are sure that cell abnormalities are usually transient in adolescent population, and they advise observation by repeating cytology or HPV DNA testing and not second level evaluation [10].

Since a quadrivalent (HPV 6, 11, 16 and 18) and a bivalent (HPV16 and 18) prophylactic HPV vaccine have been licensed, since the vaccination does not affect the course of an existing vaccine-type infection and the effectiveness of immunization for subsequent infections is uncertain, pre-vaccination data on the occurrence of HPV infection and its natural history in the adolescence population would be essential to monitor the impact of vaccination itself.

There is only one study in literature describing the age-specific distribution of HPV types within a population of girls aged 11 - 26 and it was conducted determining the specific seroprevalence [11].

It has been already demonstrated that only 50\% HPV infected women have detectable levels of antibodies to the HPV type with which they were infected and that there are specific risk factors which spoilt seropositivity as marker of HPV infection and linked pathology [12].

The aim of the present study was to describe the agespecific distribution of HPV infecting genotypes and their related lesions among females under the age of 25 , and to provide a first knowledge on Italian adolescents' HPV infection occurrence.

\section{MATERIALS AND METHODS}

\subsection{Study Population}

From September 2009 to April 2010 a prospective cohort 
study was carried out at the Colposcopy and Laser Therapy Office of Careggi University Hospital in Florence.

Recruitment included young females aged 16 - 25 years referred to our colposcopy unit after a repeated abnormal Pap smear result performed on a voluntary basis. Every patient was submitted to HPV DNA testing, colposcopic evaluation of the entire genital area and eventual bioptical sampling of unclear lesions. A brief personal medical history was collected to be informed about recurrent HPV genital lesions. Informed consent on procedures to be performed was obtained from each patient or, when under 18 of age, from her legal tutor. The study population was selected as not to be influenced to a considerable extent by demographic characteristics such as educational level, co-morbidities, smoke, oral contraceptives use, condom use. The distribution of these characteristics in our study population had to be similar to that in the general Italian female population for this group of age. Moreover the entire sampled population consisted of indigenous Italian ethnic origin.

\subsection{HPV DNA Testing}

Two cellular samples were collected from the infected genital area by scrab.

Both specimens were stored overnight at $4{ }^{\circ} \mathrm{C}$ grades until processing.

Up to five $\mathrm{mL}$ of cervical samples were centrifugated at $2000 \mathrm{~g}$ for 15 minutes at room temperature; $200 \mathrm{ul} \mathrm{of}$ the obtained cell pellets were diluted in 190 ul of digestion buffer and incubated with proteinase $\mathrm{K}$ at $56^{\circ} \mathrm{C}$ for 2 - 5 hours (EZ1 DNA Tissue kit, QIAGEN, Germany).

An automated DNA purification was performed (BioRobot EZ1, QIAGEN, Germany).

PCR (Polymerase Chain Reaction) amplification of HPV-DNA sequences was carried out by a thermocycler (MJ Research) with a two-step commercial PCR kit (BIOLINE).

First step consisted in screening the presence of HPVDNA by L1 consensus primers $(6,11,16,18,26,31,33$, 35, 39, 40, 42, 43, 44, 45, 51, 52, 53, 54, 55, 56, 57, 58, 59,66 and 68) while in a second time positive screens were typed by E6-E7 primers (6, 11 for low risk; 16, 18, 31, 33, 35, 45, 52 and 58 for high risk). The amplified products were identified by agarose gel at $2 \%$ electrophoresis and visualized by ultraviolet light. The quality of the DNA samples was validated by detection of the housekeeping gene beta-globin, as internal control.

\subsection{Management Protocol}

All cohort participants underwent a colposcopy using a $3 \%$ acetic acid solution followed by Lugol test. The colposcopic aspect was interpreted according to the International Nomenclature [13].
A cervical biopsy specimen was taken from the HPV lesion in all girls with colposcopic diagnosis of ANTZ (abnormal transformation zone) and it was analyzed in order to obtain the histological diagnosis of the lesion.

Treatment of genital warts was performed in accordance with patient's request.

Histologically proven CIN (cervical intraepithelial neoplasia) were treated by destructive or excisional laser $\mathrm{CO}_{2}$ therapy.

The choice of treatment method was based on the type of diagnosed disease. A laser vaporization therapy (destructive method) was performed for florid and flat warts, every site. Most cases of CIN 2/3 were treated by laser vaporization, in respect of patients' young age and fertility status; laser conization was reserved only to lesions extended into the endocervix (excisional method).

Laser procedures were performed by a SmartXide 50 $\mathrm{HS}$ (Deka Inc. Italia) $\mathrm{CO}_{2}$ laser with maximum power output of 50 Watt, used in super-pulsed mode at 25 Watt and connected to a Zeiss OPMI colposcope (Carl Zeiss, Oberkochen, Germany). The beam spot diameter ranged from 0.5 to $1 \mathrm{~mm}$ with an irradiance ranging from 3500 to $4000 \mathrm{~W} / \mathrm{cm}^{2}$, guided by a micromanipulator.

All treated women were checked three months after the procedure. Follow-up visits were scheduled after six months from diagnosis.

All the procedures involved in the study were performed in accordance with our Institutional guidelines and in respect of the principles of the Declaration of Helsinki.

Ethic approval for this study was obtained by the University of Florence and the AOUC teaching Hospital review board.

\subsection{Statistical Analysis}

The distribution of five characteristics of HPV-related disease (presence of HR HPV, co-infection, histologically proven high grade CIN, relapsing disease and multiple site lesions) in different age groups was evaluated analyzing variables by Fisher's Exact test as discrete ones.

\section{RESULTS}

The mean age of study population $(n=85)$ was $21.36( \pm$ 3.54 ) years; 30 patients belonged to the age group 16-19 and 55 to the age group 20 - 25 years.

Of the participants, $24.7 \%(n=21)$ had already had previous experience of HPV-related disease and had been treated by laser therapy; $61.9 \%(n=13)$ of them were younger than 20 years at the time of recurrence and $38.1 \%(n=8)$ were older than $20 ; 80.9 \%(n=17)$ of them were at the first recurrence, $14.3 \%(n=3)$ at the second one and $4.8 \%(n=1)$ at the third one. No patient 
had a previous history of CIN 2/3: all previous treatments had been performed for flat (38.4\%), mixed (15.4\%) or florid (46.2\%) warts on genital areas. Among recurrent episodes no case of CIN 2/3 occurred.

The baseline cytologic diagnosis was viral cytopathic effects in 20 cases (23.5\%), ASC-US (atypical squamous cells of undetermined significance) in 14 cases (16.5\%), LSIL (low grade squamous intraepithelial lesion) in 43 cases (50.6\%) and HSIL (high grade squamous intraepithelial lesion) in 8 cases (9.4\%). No case of ASC-H (atypical squamous cells cannot exclude HSIL) occurred during the study and in the chosen category.

By colposcopy, we diagnosed florid warts affecting a single genital area in 13 patients (15.3\%) and florid warts affecting multiple genital areas in 6 patients (7.1\%); a diagnosis of ANTZ (abnormal transformation zone) grade 1 and ANTZ grade 2 was made in 62 cases (72.9\%) and in 12 cases (14.1\%), respectively.

A cervical biopsy was performed in all cases $(n=74)$ of ANTZ in order to obtain the final histological diagnosis of the lesion and to plan the correct treatment; the biopsy showed cervical flat warts/CIN 1 in 54 cases (73\%) and CIN 2/3 in 20 cases (27\%).

Viral genotyping showed 42 (49.4\%) single HR (high risk) infections, 31 (36.5\%) single LR (low risk) infections and 12 (14.1\%) co-infections. In 11 cases (91.7\%) of co-infection both infecting viruses were high risk types, in one case there was a co-infection with two low risk types. There was no reported case of co-infection with a LR genotype and a HR one.

Seventeen HPV types were detected in the study cohort. The most common HPV type was HPV-6 (overall prevalence 15.3\%), followed by HPV-16 (14.3\%), HPV11 (12.24\%), HPV-56 (9.18\%), HPV-31 (7.14\%), HPV18 and HPV-52 (6.12\%), HPV-33 (5.1\%), HPV-51 (4.1\%), HPV-54 and HPV-45 (8.4\%), HPV-58 and HPV68 (3.06\%), HPV-35 and HPV-72 (2.04\%), HPV-53 and HPV-73 (1.02\%).

We observed a predominance of low risk infections among 16-19-year-old girls: the overall prevalence of LR HPV amounted to $80 \%$ in this age group $(83.3 \%$ and 79.2\% among 16 - 17 and 18-19-year- old girls, respectively). On the contrary, we found a predominance of high risk infections among 20-25-year-old females: the overall prevalence of HR HPV was $85.5 \%$ in this age group (72.7\%, 89.5\% and 88\% among 20 - 21, 22 - 23 and 24 - 25-year-old girls, respectively).

The univariate analysis of chosen characteristics of HPV-disease demonstrates the statistically significative difference of this infection between the two groups of age (Table 1).

All participants demanded for treatment by destructive
Table 1. Age-stratified characteristics of HPV-related disease.

\begin{tabular}{cccc}
\hline & $\begin{array}{c}\text { Age } 16-19 \\
(30 \mathrm{pts})\end{array}$ & $\begin{array}{c}\text { Age } 20-25 \\
(55 \mathrm{pts})\end{array}$ & $\mathrm{P}<0.005$ \\
\hline HR HPV & 6 & 47 & $<0.0001$ \\
CO & 1 & 11 & 0.04 \\
INFECTION & 0 & 20 & $<0.0001$ \\
CIN 2/3 & 0 & 9 & $<0.0001$ \\
DISEASE & 17 & 2 & $<0.0001$ \\
RELAPSE & 13 & & \\
MULTI SITE & 13 & & \\
DISEASE & & &
\end{tabular}

or excisional physical therapy in our clinic. A laser vaporization was performed in 81 patients: $23.4 \%(n=19)$ of them had perineal florid or mixed warts, $56.8 \%(n=46)$ had cervical flat warts and $19.8 \%(n=16)$ had a diagnosis of CIN 2/3. In four cases of high grade CIN (two CIN 2 and two CIN 3) with not visible squamo-columnar junction (SCJ) a laser conization was preferred.

All patients were negative at the cyto-colposcopic examination performed three months after treatment.

\section{DISCUSSION}

To our present knowledge this is the first study providing the age-specific distribution of HPV types within mucosal samples of such a young population.

The common thought of HPV in this age group is that the infections result in frequent abnormal cytology not correlated to histological proven lesions, most of them are transient, with frequent clearance of HPV and of the cytological alteration itself. Thus current strategies for adolescents with abnormal cytology include conservative management, avoiding invasive procedures [14].

All patients included in our observational study were young sexually active females who started to make cervical screening by their own will. This is a common actual practice, thanks to screening campaigns, women are sensitive to cervical cancer prevention and often send their sexually active daughters to such examinations.

The present study relevance, whether influenced by a small sample size, is linked to show the real burden that HPV-linked pathologies may have on youth.

As we observed, HPV-related disease heavily manifests also under the age of 25 , which is the initial term for Italian cervical screening.

Girls under the age of 20 years have a low frequency of HR HPV infections, a low incidence of co-infections and not-existent incidence of high grade cervical intraepithelial neoplasia, but they experiment a disease affecting multiple genital areas simultaneously (43.3\%) and tending to relapse frequently (43.3\%), thus the volunteer request of treatment.

Moreover $85.5 \%$ of girls over 20 years of age experiment the grade of disease that is generally coupled to 
older women, because principally infected by HR HPV.

On the basis of these data we are able to observe that the initial age of the actual cervical cytological screening program in Italy seems to be much older than the starting of the HPV linked pathology within the young population, determining a loss rate of CIN, which are part of the discussed failure of national cervical cancer screening programs together with never reached women and patients loss at follow-up.

Within the study group, among the girls aging between 16 and 19 years old, we observed that $70 \%$ of diagnosed CIN 1 were associated with florid genital warts. This association makes the HPV-linked pathology of the youngest a clinically manifested disease in the majority of the cases, thus not requiring institutionalized screening or diagnostic programs. Otherwise, in our study group, girls of 20 - 25 years of age were affected by cervical isolated HPV-linked lesions in $96.4 \%$ of the cases, without external clinical manifestations of the infection. Moreover, as previously said, these girls are the ones prevalently infected by HR HPV, suffering by CIN 2/3.

This observation may suggest the inclusion in the Italian cervical cancer screening programs of girls aging 20 - 24, as other worldwide countries already did [15].

The particular stratification of HPV genotypes in different age groups that this study shows and the cut-off of incidence of LR HPV in the girls older than 20 suggest further considerations.

Contemporary literature already explained the role of humoral and cellular immune response towards HPV infections. There are many observations that demonstrate how antibodies are failing in the protection against HR $\mathrm{HPV}$ and their consequent disease. One of this is the observation that only $50 \%$ of HR genotypes infected women have circulating antibodies against HPV and moreover that the circulating HPV-antibody level is as higher as more severe the cervical lesion is [12]. Furthermore is already well known how low CD4 count HIV positive patients are at major risk for cervical dysplasia than normal CD4 count ones, demonstrating how the cellular immune response is fundamental as protection against HR HPV linked disease [16].

After the introduction of HPV quadrivalent vaccine in Australia, a relevant reduction of HPV linked pathology rate among women younger than 28 years was observed, while the prevalence of HPV-linked disease remained stable among older women. Benign lesions (cervical, vulvar or other genital warts) were reduced most with substantial stability of CIN 2/3 prevalence in the population [17].

Present data support this observation in suggesting that antibody protection, natural or vaccine inducted, best expressed towards low risk HPV linked pathology, could be one of the hypothetical explanation of such a stratification of HPV genotypes within different groups of age.

Another hypothesis bears from a recent study on biological differences in mucosal immune function which were evacuated to play a fundamental role in the increate vulnerability of adolescents compared to adults to sexually transmitted infections [18].

This study evaluated in healthy young women the association between their type of cervical epithelium and the levels of inflammatory and regulatory cytokines in their mucus. The results of this study show that inflammatory cytokines are significantly higher in the immature group compared to the mature one.

The hypothesis is that a higher cytokine profile at the columnar epithelium mounts to protect the columnar epithelium which is only single layered and more prone to proliferative infections such as LR HPV, until a prompt immune response is evocated and able to clear them. At the same time this higher cytokine profile could foreshadow a tendency towards a more severe inflammatory response that leads to harmful tissue damage such as multisite HPV lesions.

These data on reactive inflammatory response of adolescent cervical epithelium are produced only on healthy non-infected women and implications for infections derive only from speculative association between them and the present data of an existent particular age-specific distribution of different HPV genotypes.

Perspectives for Authors are to plan further studies for understanding the role of cervical epithelial type in acquisition of HPV infection and the consequent immune response during active infection.

\section{REFERENCES}

[1] Lenselink, C.H., Melchers, W.J.G., Quint, W.G.V., et al. (2008) Sexual behaviour and hpv infections in 18 to 29 year old women in the prevaccine era in the Netherlands. PLOS ONE, 3, e3743. doi:10.1371/journal.pone.0003743

[2] Burchell, A.N., Richardson, H., Mahmud, S.M., et al. (2006) Modeling the sexual transmissibility of human papillomavirus infection using stochastic computer simulation and empirical data from a cohort study of young women in Montreal, Canada. American Journal of Epidemiology, 163, 534-543. doi:10.1093/aje/kwj077

[3] Weinstock, H., Berman, S. and Cates, W. J. (2004) Sexually transmitted diseases among American youth: Incidence and Prevalence Estimates, 2000. Perspect Sex Reprod Health, 36, 6-10. doi:10.1363/3600604

[4] Forhan, S.E., Gottlieb, S.L., Sternberg, M.R., et al. (2009) Prevalence of sexually transmitted infections among female adolescents aged 14 to 19 in the United States. Pediatrics, 124, 1505-1512. doi:10.1542/peds.2009-0674

[5] Barthell, E., Woelber, L., Hellner, K., et al. (2009) Baseline characteristics and prevalence of HPV 6, 11, 16, 18 in young German women participating in phase III 
clinical trials of a quadrivalent HPV (6/11/16/18) vaccine. Archives Gynecology and Obstetrics, 279, 803-807. doi:10.1007/s00404-008-0806-1

[6] Manhart, L.E., Holmes, K.K., Koutsky, L.A., et al. (2006) Human papillomavirus infection among sexually active young women in the United States: Implications for Developing a Vaccination Strategy. Sexually Transmitted Diseases, 33, 502-508. doi:10.1097/01.olq.0000204545.89516.0a

[7] Ho, G.Y.F., Bierman, R., Beardsley, L., Chang, C.J. and Burk, R.D. (1998) Natural history of cervicovaginal papillomavirus infection in young women. The New England Journal of Medicine, 338, 423-428. doi:10.1056/NEJM199802123380703

[8] De Sanjosé, S., Diaz, M., Castellsagué, X., et al. (2007) Worldwide prevalence and genotype distribution of cervical human papillomavirus DNA in women with normal cytology: A meta-analysis. The Lancet Infectious Diseases, 7, 453-459. doi:10.1016/S1473-3099(07)70158-5

[9] Confortini, M., Carozzi, F., Zappa, M., et al. (2010) Human papillomavirus infection and risk factors in a cohort of Tuscan women aged 18-24: Results at recruitment. BMC Infectious Diseases, 10, 157-167. doi:10.1186/1471-2334-10-157

[10] Moscicki, A.B., Shiboski, S., Hills, N.K., et al. (2004) Regression of low-grade squamous intra-epithelial lesions in young women. Lancet, 364, 1678-1683. doi:10.1016/S0140-6736(04)17354-6

[11] Kramer, M., Mollema, L., Smits, G., Boot, H., de Melker, H. and Van Der Klis, F. (2010) Age-specific HPV seroprevalence among young females in the Netherlands. Sexually Transmitted Diseases, 86, 494-499. doi:10.1136/sti.2009.041210

[12] Porras, C., Bennett, C., Safaeian, M., et al. (2010) Costa Rica HPV Vaccine Trial (CVT) Group. Determinants of seropositivity among HPV-16/18 DNA positive young women. BMC Infectious Diseases, 10, 238.

[13] Stafl, A. and Wilbanks, G.D. (1991) An international terminology of colposcopy: Report of the nomenclature committee of the international federation of cervical pathology and colposcopy. Obstetrics and Gynecology, 77, 313-314. doi:10.1097/00006250-199102000-00032

[14] Wright, T.C. Jr., Massad, L.S., Dunton, C.J., et al. (2007) 2006 consensus guidelines for the management of women with abnormal cervical cancer screening tests. American Journal of Obstetrics and Gynecology, 197, 346-355. doi:10.1016/j.ajog.2007.07.047

[15] Anttila, A., von Karsa, L., Aasmaa, A., et al. (2009) Cervical cancer screening policies and coverage in Europe. European Journal of Cancer, 45, 2649-2658. doi:10.1016/j.ejca.2009.07.020

[16] Harris, T.G., Burk, R.D., Palefsky, J.M., et al. (2005) Incidence of cervical squamous intraepithelial lesions associated with HIV serostatus, CD4 cell counts, and human papillomavirus test results. Journal of the American Medicine Association, 293, 1471-1476. doi:10.1001/jama.293.12.1471

[17] Fairley, C.K., Hocking, J.S., Gurrin, L.C., Chen, M.Y., Donovan, B. and Bradshaw, C.S. (2009) Rapid decline in presentations of genital warts after the implementation of a national quadrivalent human papillomavirus vaccination programme for young women. Sexually Transmitted Diseases, 85, 499-502. doi:10.1136/sti.2009.037788

[18] Hwang, L.Y., Scott, M.E., Ma, Y. and Moscicki, A.B. (2011) Higher levels of cervicovaginal inflammatory and regulatory cytokines and chemokines in healthy young women with immature cervical epithelium. Journal of Reproductive Immunology, 88, 66-71. doi:10.1016/j.jri.2010.07.008 